\title{
Deceleration in the radio jets of GRS 1915+105?
}

\section{James Miller-Jones, ${ }^{*}{ }^{a}$ Michael Rupen, ${ }^{b}$ Rob Fender, ${ }^{a c}$ Tony Rushton, ${ }^{d}$ Guy Pooley ${ }^{e}$ and Ralph Spencer ${ }^{d}$}

aAstronomical Institute 'Anton Pannekoek', University of Amsterdam, Kruislaan 403, 1098 SJ, Amsterdam, the Netherlands

${ }^{b}$ NRAO, Array Operations Center, 1003 Lopezville Road, Socorro, NM 87801, USA

${ }^{c}$ University of Southampton, Highfield, Southampton, SO17 1BJ, UK

${ }^{d}$ University of Manchester, Jodrell Bank Observatory, Cheshire, SK11 9DL, UK

${ }^{e}$ Astrophysics, Cavendish Laboratory, J. J. Thomson Avenue, Cambridge CB3 OHE, UK

E-mail: jmillerescience.uva.nl, mrupeneaoc.nrao.edu,

rpfephys.soton.ac.uk arushton@jb.man.ac.uk, guy@mrao.cam.ac.uk,

resejb.man.ac.uk

There is currently a discrepancy in the proper motions measured on different angular scales in the approaching radio jets of the black hole X-ray binary GRS 1915+105. Lower velocities were measured with the VLA prior to 1996 than were subsequently found from higher-resolution observations made with the VLBA and MERLIN arrays. We initiated an observing campaign to use all three arrays to track the motion of the jet knots from the February 2006 outburst of the source, giving us unprecedented simultaneous coverage of all angular scales, unbroken from scales of 0.8 milliarcseconds out to 20 arcseconds. We measured a proper motion of $16.6 \mathrm{mas} \mathrm{d}^{-1}$, demonstrating that there has been no significant permanent change in the properties of the jet since 1994 . We found no conclusive evidence for deceleration of the jet knot, unless this occurs within 75 mas of the core.

VI Microquasar Workshop: Microquasars and Beyond September 18-22, 2006

Como, Italy

\footnotetext{
* Speaker.
} 


\section{Introduction}

Since they were first observed [12], the radio jets in the black hole X-ray binary GRS 1915+105 have been the focus of intensive study. The proper motions initially measured with the Very Large Array (VLA) [12] were $\mu_{\text {app }}=17.6 \pm 0.4$ mas d $^{-1}$ for the approaching (southeastern) jet and $\mu_{\text {rec }}=9.0 \pm 0.1$ mas d $^{-1}$ for the receding (northwestern) counterjet. Fairly consistent values were measured with the VLA for other ejection events [13], and no evidence was seen for any deceleration of the jet knots, which were always seen to move ballistically outwards from the core. However, observations of a 1997 ejection event with the Multi-Element Radio Linked Interferometer Network (MERLIN) [6] measured significantly higher proper motions, $\mu_{\text {app }}=23.6 \pm 0.5$ mas d $^{-1}$ and $\mu_{\text {rec }}=10.0 \pm 0.5$ mas d $^{-1}$, albeit on smaller angular scales. Again, the jet knots were observed to move ballistically. Such high proper motions were also confirmed by observations with the Very Long Baseline Array (VLBA) [ [] and further MERLIN observations [10]. In no case was there ever any evidence for deceleration. Fig. 11 shows all the published proper motions for the jets in this system as a function of time, and highlights the stark discrepancy between observations prior to 1997 made with the VLA and those made later with higher-resolution arrays.

This discrepancy in the proper motions of the jet knots between milliarcsecond and arcsecond angular scales has important implications for the properties of the system. Measuring the proper motions gives an upper limit to the source distance, assuming the jets are intrinsically symmetric on either side of the core, and allows the derivation of the jet speed and inclination angle to the line of sight [12]. This can then be used to deduce the jet Lorentz factor and, together with the peak brightness and rise time of the ejection event, can be used to constrain the minimum energy and the equipartition magnetic field of the jet (e.g. [6]). Furthermore, deceleration has been definitively observed in very few sources, XTE J1550-564 being the best example to date [9]. If this were the explanation for the discrepancy, it would place fairly significant constraints on the jet power and the density of the surrounding interstellar medium (ISM).

Several possibilities have been put forward to explain the apparent discrepancy. Precession causing a change in the angle to the line of sight of an otherwise physically identical jet cannot explain the observations [6]. The jet velocities could be intrinsically different at different epochs. Alternatively, resolution effects could be at work between the different arrays [8], leading to a blending of components. If the ejecta decreased sequentially more rapidly in flux density with increasing distance from the core, beam smearing effects could reduce the proper motions measured at low resolution. The knots could simply be decelerating as they moved outwards, although the ballistic motions of the observed knots argues against this. Alternatively, there could have been an intrinsic change in the system between the time when the VLA observations were taken (19941995) and the dates of the higher-resolution observations (1997-2001) [10]. Since the source was not detected until 1992 [3], despite existing X-ray satellites prior to that time having the sensitivity to detect it, the system might only then have emerged from quiescence. Indeed, it has been suggested [14] that the system has been in outburst since 1992, and may soon switch off again, moving back into quiescence. In this case, the jets might since 1992 have evacuated a cavity through which the ejecta could propagate, such that they could coast for longer during later outbursts, giving rise to the higher proper motions measured with the VLBA and MERLIN. To try to discern the true cause of the proper motion discrepancy, we proposed to track the motion of the ejecta in GRS 1915+105 


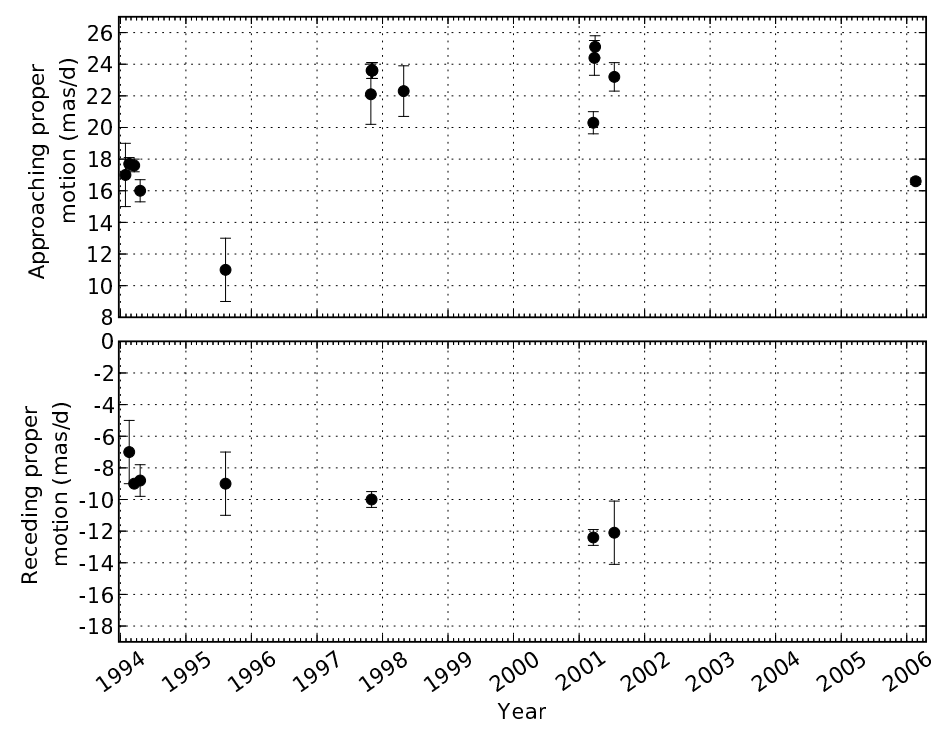

Figure 1: Measured proper motions in GRS 1915+105, as a function of time. The August 1995 point is based on only 2 epochs of data, so is probably less robust.

over all available angular scales, using the VLBA, MERLIN and also the VLA.

\section{Observations and data reduction}

We triggered our Target of Opportunity proposal [11] after being alerted to a radio flare in GRS $1915+105$ by the RATAN-600 monitoring programme (S. Trushkin, private communication). The Rossi X-ray Timing Explorer (RXTE) All Sky Monitor (ASM) data showed that this coincided with a significant $X$-ray flare, giving us confidence that an ejection event was underway. We obtained four epochs of VLBA observations, three of MERLIN observations, and quasi-weekly monitoring with the VLA until the end of its A-configuration trimester. A montage of all the observations during the early period when all three arrays were taking data is shown in Fig. 2.

\subsection{VLBA observations}

The VLBA observations were made in parallel at two different frequencies, 8.42 and $2.27 \mathrm{GHz}$. Observations were taken using dual polarisation, 2-bit sampling, with sixteen 500-kHz channels giving a total bandwidth of $8 \mathrm{MHz}$ at each frequency. Owing to scatter-broadening of GRS 1915+105 [П, we were forced to discard the longest baselines, slightly compromising our resolution.

Only in the first of the four VLBA epochs was the original southeastern component seen which corresponded to the knot monitored with the VLA. Its separation agreed well with that seen at the VLA on the same day (MJD 53794). Both the core and the ejecta appeared slightly elongated, and at both frequencies there seemed to be a hint of a receding northwestern component. By the second epoch (MJD 53798), the southeastern component had faded and was no longer detectable, although there is evidence that at least two new sets of knots had been ejected. In the $8.4 \mathrm{GHz}$ image, both 

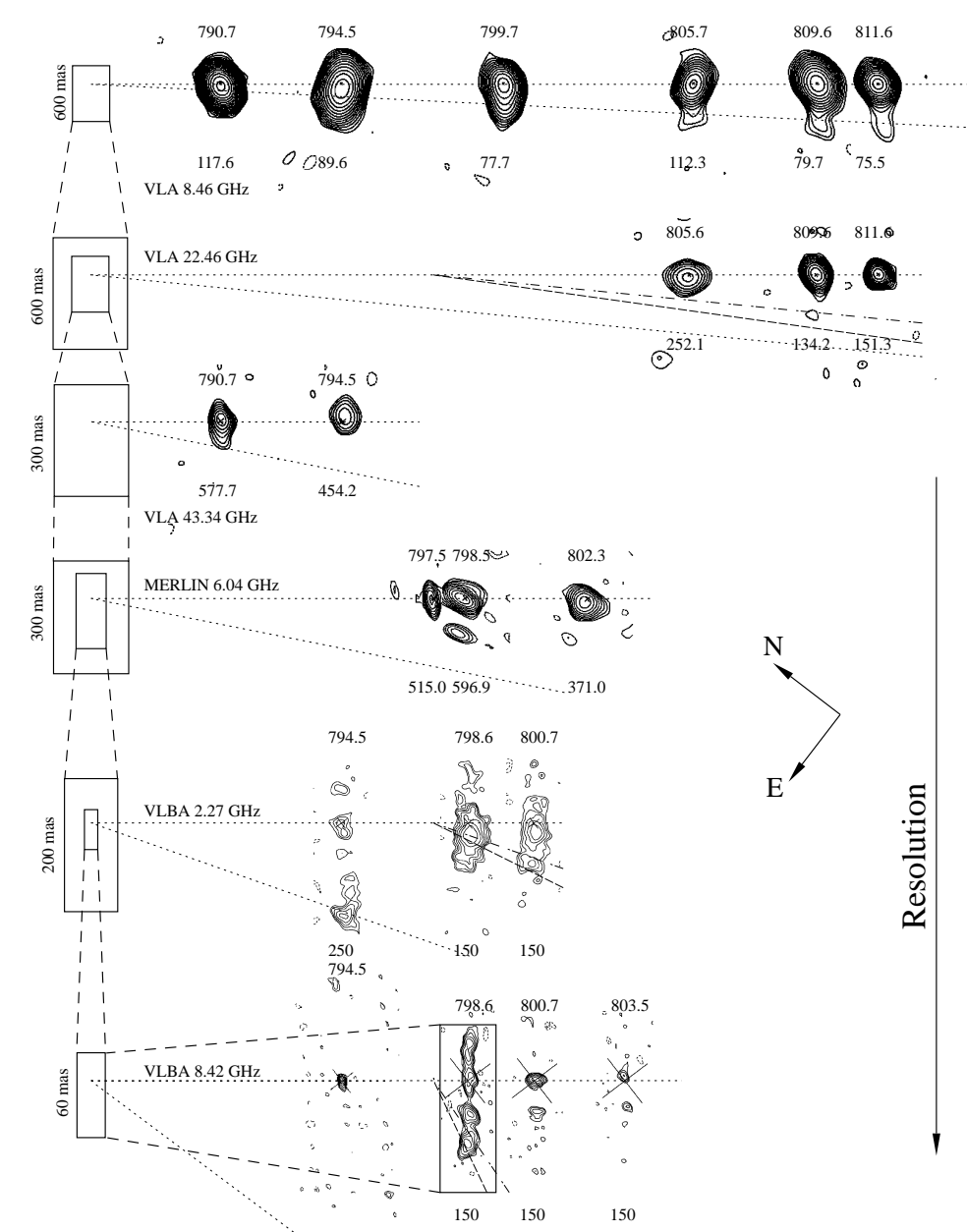

Q.

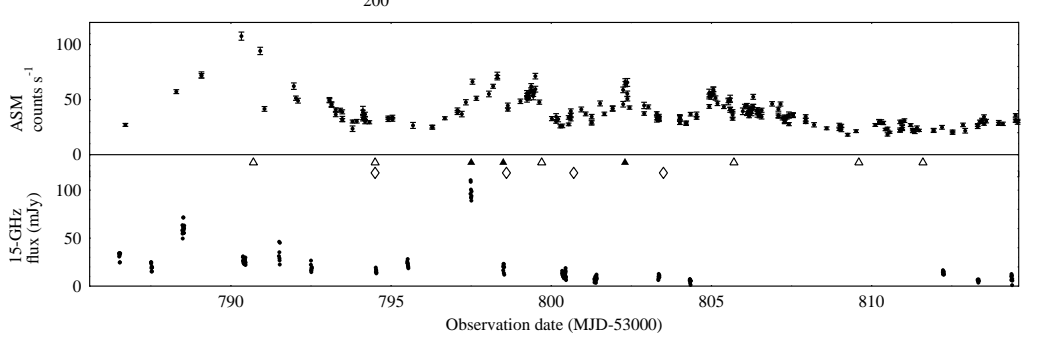

Figure 2: Radio images from our monitoring campaign. 15-GHz flux densities from the Ryle Telescope and the RXTE ASM 1.5-12 keV X-ray count rates are shown in the bottom plots. The times of the observations are shown at the top of the 15-GHz plot (open triangles for the VLA, diamonds for the VLBA, and filled triangles for MERLIN). Resolution improves downwards, showing, in order, VLA $8.46 \mathrm{GHz}$ (top), $22.46 \mathrm{GHz}$, and $43.34 \mathrm{GHz}$ images, MERLIN $6.03 \mathrm{GHz}$ images, VLBA $2.27 \mathrm{GHz}$, and finally VLBA $8.42 \mathrm{GHz}$ images (bottom; note the different scale for the first epoch). Observation dates (MJD - 53000) are shown above each image, and the image rms $\left(\mu \mathrm{Jy} \mathrm{bm}^{-1}\right)$ below. Contours are at $\pm(\sqrt{2})^{n} \sigma$, where $n=3,4,5, \ldots$. The cross shows the VLBA core. All images have been rotated $37.5^{\circ}$ anticlockwise. Dotted lines show the fitted proper motion of $16.6 \mathrm{mas} \mathrm{d}^{-1}$ from the initial ejection event, and the dashed and dot-dashed lines show nominal proper motions of 23.6 and 16.6 mas d $^{-1}$ for a later ejection event. 
the approaching and receding components were seen, and the core component appeared marginally elongated, hinting at an even more recent ejection event.

Two days later, at MJD 53800, these new components had also faded at $8.4 \mathrm{GHz}$, leaving an unresolved core and a new, resolved southeastern component. At $2.3 \mathrm{GHz}$ however, the source was clearly resolved, and a component presumably corresponding to the knots from the previous epoch could be seen. In the last epoch, MJD 53803, the core was again unresolved and a very weak southeastern component was present at $8.4 \mathrm{GHz}$. For the known range of proper motions of the ejecta in this system, the approaching component in this image cannot be related to those seen in the previous two images. No source was detected at $2.3 \mathrm{GHz}$. For this observing run, we were missing two of the antennas in the southwestern United States (Fort Davies and Pie Town), which removed several of the short baselines in the array. Coupled with the loss of the long baselines to scattering, this rather compromised the $u v$-coverage, possibly explaining the non-detection.

\subsection{MERLIN observations}

For the MERLIN observations, the observing frequency was $6.0353 \mathrm{GHz}$ with a bandwidth of $14 \mathrm{MHz}$. At each epoch, observations were also made of the flux and polarisation angle calibrator 3C 286, the point source calibrator OQ 208, and the phase reference source, B 1919+086. The observations were made using the five outstations and the Mark 2 antenna at Jodrell Bank. The MERLIN d-programs were used to perform initial data editing and amplitude calibration, and the data were then imported into the National Radio Astronomy Observatory's (NRAO) Astronomical Image Processing System (AIPS) software package for further data reduction. The MERLIN pipeline was then used to image and self-calibrate the phase reference source, and apply the derived corrections to GRS 1915+105, which was subjected to further iterations of phase-only self-calibration.

The first MERLIN epoch caught the start of a flare, seen to begin at MJD 53797.465 \pm 0.003 . The variable amplitude meant that the last few hours of observation had to be flagged to prevent the violation of the basic assumption of synthesis imaging that the source structure should not change during the observation. Similarly, the second epoch of observation caught the decay of a further flare, dropping from 107 to $20 \mathrm{mJy}$ over the course of $6.5 \mathrm{~h}$. Nevertheless, imaging that epoch showed both the core component and a southeastern jet knot. The final epoch appeared to show only an unresolved core.

\subsection{VLA observations}

The VLA was in A-configuration, and 1-hour observations were made on a quasi-weekly basis. The main observing frequency was $8.4 \mathrm{GHz}$, although initially when the source was bright, higher frequencies were also used in an attempt to improve the resolution. As the ejecta moved outwards and faded below detectability at $8.4 \mathrm{GHz}$, lower frequencies were used in order to take advantage of the assumed steep spectrum $\left(v^{-0.6}\right)$ of the jet knots to aid in their detection. We used 3C 48 as the flux calibrator and J $1924+156$ as the phase calibrator. The fast switching mode was used at frequencies above $5 \mathrm{GHz}$ in order to reduce the slew time between the secondary calibrator and the target while cycling between the two fast enough to account for tropospheric phase variations. This allowed for diffraction-limited imaging at high frequencies in the case that the source was not strong enough for self-calibration to work reliably. Data reduction was performed using standard procedures within AIPS. 

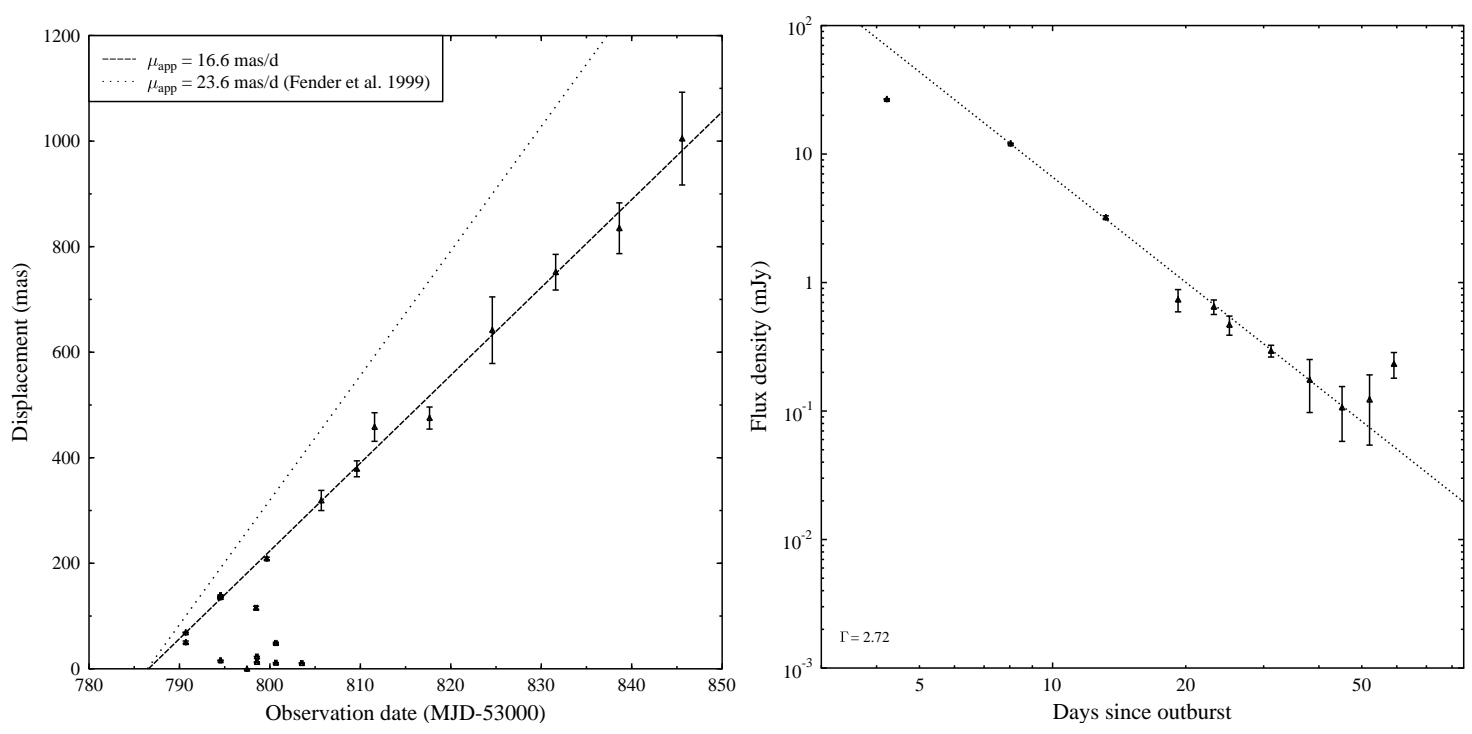

Figure 3: Left: Measured angular separations from the core of the SE component ejected on MJD 53786. The separations measured with the VLBA and MERLIN are also included, which form the scatter of points at very low angular separations between MJD 53794 and 53803. Right: Measured VLA flux densities of the approaching jet knot ejected on MJD 53786, with a best-fitting line corresponding to a power-law decay of index 2.72 overlaid.

We tracked a single ejected component for $59 \mathrm{~d}$ as it moved southeast, out to an angular separation of almost 1 arcsec. The ejection event appeared to be one-sided, since only on MJD 53817 did we see any evidence for a receding component. Tracking the ejecta over such a long period of time gave extremely good constraints on its proper motion, which we fitted as $16.6 \pm 0.2$ mas d $^{-1}$. This fit (to the VLA proper motions only) is shown in Fig. 3. It is clearly incompatible with the previously-measured MERLIN proper motions [6], and there is no evidence for any deceleration. To verify this, we fitted the angular separations with both a straight-line fit (ballistic motion) and a quadratic fit (corresponding to deceleration), and performed an F-test. This gave a value $F_{1,10}=0.34$, implying that the quadratic fit was not significantly better, i.e. there was no significant evidence for any deceleration of the ejecta on the angular scales probed by the VLA. The flux density of the ejecta showed a power-law decay with time, $S=S_{0}\left[\left(t-t_{0}\right) / \text { days }\right]^{-2.72}$ (Fig. 3). The lack of any rebrightening argues against any deceleration due to an interaction with the ISM.

\section{Discussion}

The fact that we have measured the same proper motion (to within errors) as seen with the VLA in 1994 would seem to rule out the secular change scenario [10]. If the radio jets had only switched on in 1992 and subsequently evacuated a cavity in the ISM, allowing the jets to propagate further at high velocity before decelerating, then we should have measured a high proper motion, of order 23.6 mas d $^{-1}$, with our VLA data.

The VLBA data would suggest that resolution effects are not responsible for the discrepancy between the proper motions measured on large and small angular scales. As well as the range of 
angular separations measured by MERLIN and the VLA overlapping, the image of MJD 53794 shows only a single VLBA component, rather than a string of successive knots. Convolving the VLBA images on MJD 53794 (the only day with both VLA and VLBA observations) to the VLA resolution gives images consistent with those seen by the VLA, further strengthening the argument against resolution effects causing the proper motion discrepancy.

From our measured proper motions, and the values quoted in the literature [12, 6, 13, 4, 10], it would appear that GRS 1915+105 is capable of producing ejecta with a range of proper motions. In no case has there ever been any evidence for deceleration. Thus, if the spread of proper motions is intrinsic, this would imply that the speeds of the jet knots are not fixed by the properties of the black hole powering the jets (its mass or spin). Variation in the speed of the ejecta is a feature of the "unified model", whereby the Lorentz factor of the jets increases as the X-ray spectrum softens in the Very High State, leading to internal shocks within the flow which are seen as discrete ejecta [5]. For those discrete ejecta to have different observed proper motions would then imply a variable velocity discrepancy between the jet Lorentz factor in the hard and quiescent states, and that in the soft state. Since the inner edge of the accretion disc is believed to move inwards during this transition between the hard and soft states, this would imply a different inner disc radius for each outburst. This is certainly plausible.

There is in fact no strong evidence for the jets in any X-ray binary to have identical proper motions during different outbursts. The number of systems in which radio proper motions from multiple outbursts have been measured is small (GRS 1915+105, Cygnus X-3, GRO J 1655-40, and SS 433). SS 433 has a roughly constant jet velocity of $\sim 0.26 c$, but shows a standard deviation of 0.013 in $\beta$ [2]. Thus while a varying proper motion in GRS 1915+105 would not necessarily be unusual, it seems highly coincidental that if the observed speeds are drawn from some underlying distribution, there should be such a clear distinction between the high proper motions observed only by the high-resolution arrays, and the lower values seen only with the VLA (see Fig. (4). At present however, we are still in the regime of small number statistics. Nevertheless, a KolmogorovSmirnov test gave a probability of $1.78 \times 10^{-4}$ that the VLA and higher-resolution observations were drawn from the same distribution.

Since we were unable to observe the ejecta from the initial event with the VLBA and MERLIN, we cannot definitively rule out the possibility that the ejecta decelerated on angular scales $<$ 100 mas, before the VLA was able to accurately resolve them. In fact, extrapolating the proper motion measured with the VLA back to zero angular separation gives an ejection date of MJD 53786.57, $1.94 \mathrm{~d}$ prior to the peak of the $15-\mathrm{GHz}$ radio flare detected with the Ryle Telescope. While the radio flare is in fact the latest possible date of ejection, and the true ejection date could have been somewhat earlier, this is suggestive of deceleration between the radio flare and the first set of VLA observations. Assuming an initial proper motion of $23.6 \mathrm{mas} \mathrm{d}^{-1}$ and an ejection date corresponding to the time of the 15-GHz flare, then extrapolating the best fitting line for the VLA proper motion backwards in time implies that the deceleration should be occurring on angular scales of $\sim 75$ mas from the core. But previous MERLIN observations [6, 10] tracked the ejecta out to angular scales of $>300$ mas, with no compelling evidence for deceleration. Fig. 5 shows how the angular separations changed with time since ejection for all the events reported in the literature (using the derived ejection dates). This shows no clear evidence for deceleration at any specific distance from the core. The diagram shows two clear tracks for the approaching jet, one sampled 


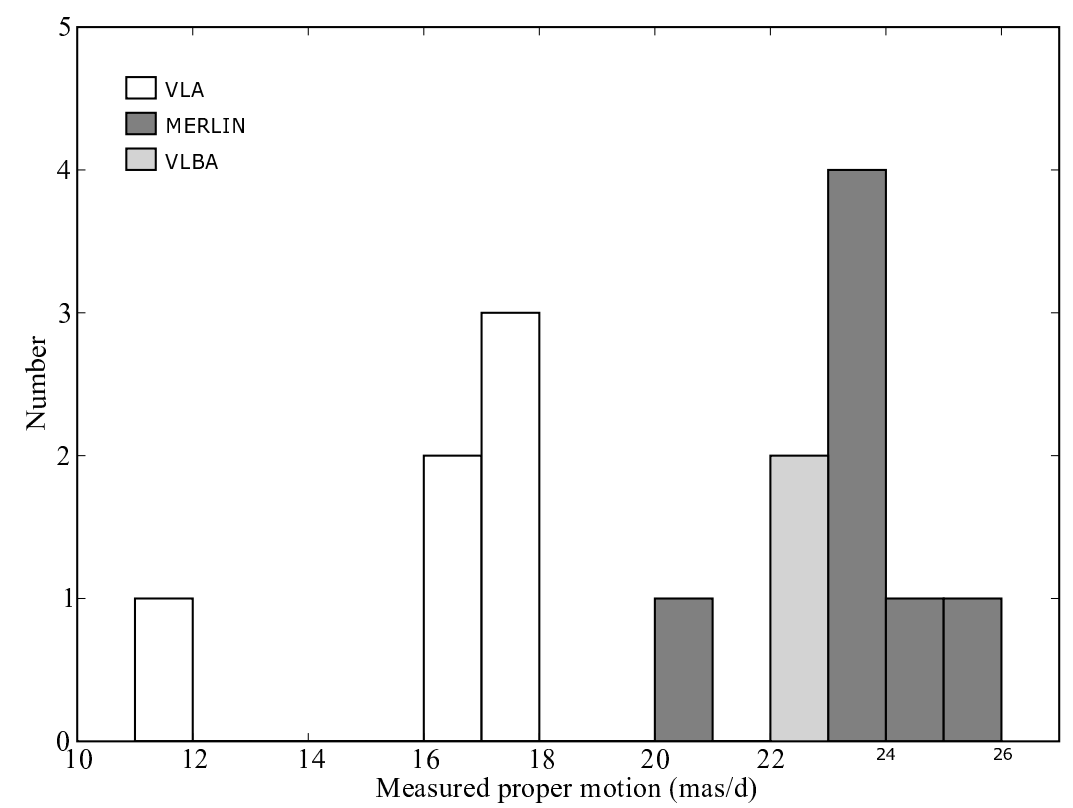

Figure 4: Histogram of the measured proper motions [13, 6, 10, \#] of the approaching jet knot of GRS $1915+105$, colour-coded by array. The measurement of $11 \pm 2$ mas d $^{-1}$ is considered unreliable, since the jets were resolved in only one image.

by the higher-resolution arrays, and the other, with lower proper motions, taken with the VLA.

Circumstantial evidence for deceleration on small scales in GRS 1915+105 can be seen in the linear polarisation 'rotator event' observed in January 2001 [ []. The electric vector position angles measured with the Australia Telescope Compact Array (ATCA) at 4.80 and $8.64 \mathrm{GHz}$ rotated smoothly together through $50^{\circ}$ over the course of $\sim 0.2 \mathrm{~d}$. The constant separation between the position angles at the two frequencies ruled out Faraday rotation, and was attributed to a changing magnetic field orientation. We note however that an accelerating (or decelerating) jet would show a swing in polarisation position angle as the aberration angle varied [1]. If this was the true explanation for the 'rotator event', and we attribute the detected polarisation to the southeastern (approaching) component (as seen by [6, 10]), which we assume to have been ejected at the time of the spike in the RXTE data [才], then this deceleration would have been occurring somewhere between 0.2 and $1.0 \mathrm{~d}$ after ejection. Such an effect was not seen in the ATCA observations of the March 2001 ejection event, taken between 1.5 and $1.9 \mathrm{~d}$ after the zero-separation date [10]. This would imply that if all outbursts followed the same pattern, deceleration would have to occur within $\sim 50$ mas. However, this evidence remains circumstantial, and would appear to be at odds with the MERLIN points $8-13 \mathrm{~d}$ after ejection on the $23.6 \mathrm{mas} \mathrm{d}^{-1}$ track in the inset to Fig. 5 .

Ultimately, the evidence for deceleration in GRS $1915+105$ is not conclusive. It is perhaps most likely that the jet speed can vary, although the VLA and higher-resolution proper motion measurements do not appear to be drawn from the same distribution. More observations are certainly needed to verify this hypothesis, since we are still in the regime of small number statistics. 


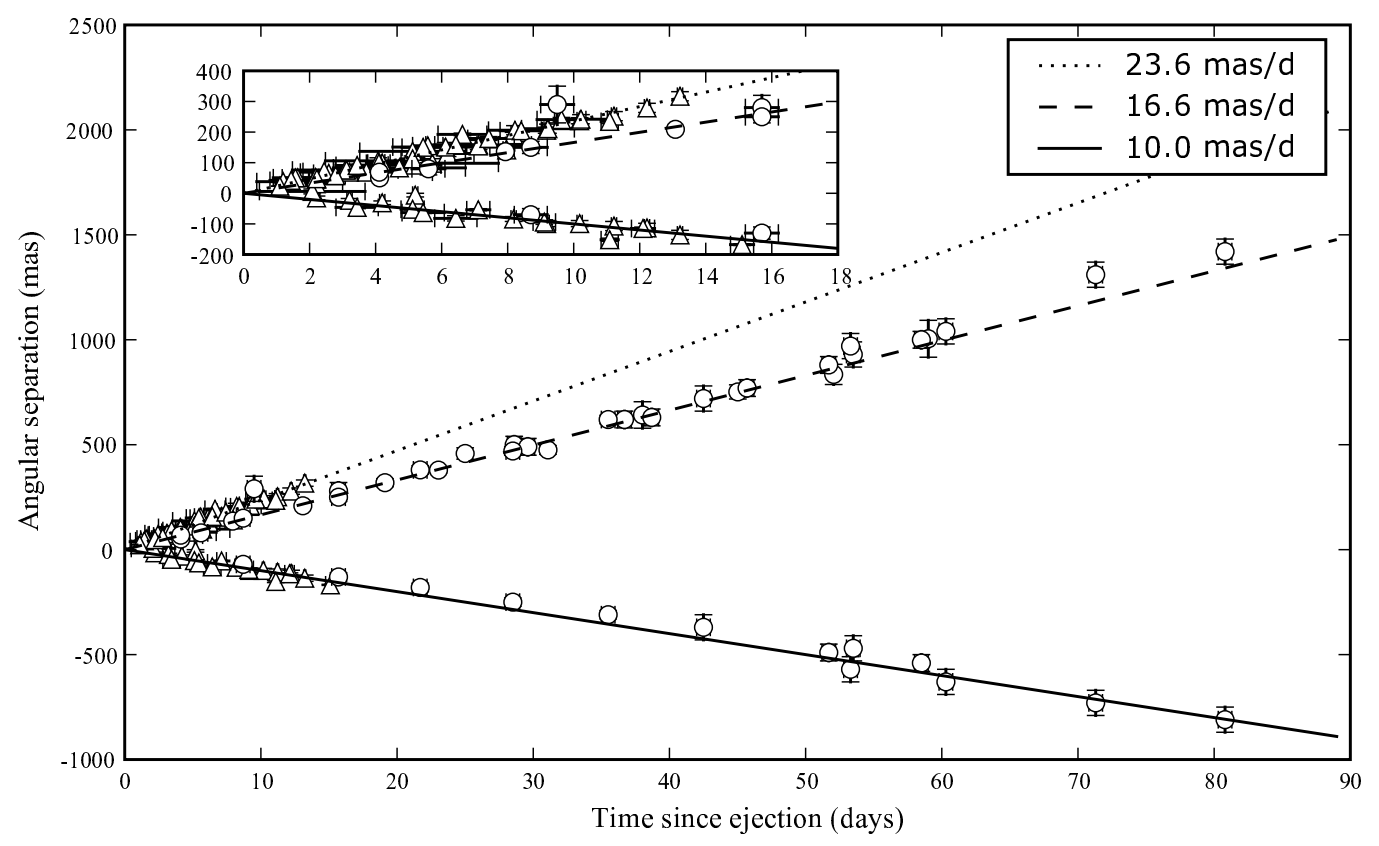

Figure 5: Variation in measured angular separation with time since ejection. Data taken from VLA [13], MERLIN [6, 10] and VLBA [П] observations of GRS 1915+105. Ejection dates were taken from the ballistic motion fits reported in the source literature. Open circles show VLA data, open triangles show VLBA or MERLIN data. The inset shows a zoomed-in version of the smallest angular scales. There is no clear evidence for deceleration on any angular scale.

\section{Conclusions}

We measured the proper motion of a jet knot ejected during the February 2006 outburst of GRS $1915+105$ as 16.6 mas d $^{-1}$. This is the first measurement of such a low proper motion since 1995, and shows that there has been no secular change in the system which would allow the jet knots to propagate at a faster speed. The VLBA images showed that although the first ejection was the brightest, there were in fact several ejection events over the course of two weeks. The brightest jet knot shows no evidence for deceleration on angular scales greater than 100 mas, and its flux density decays as a simple power law between 8 and 45 days after the ejection event. Simultaneous VLA and VLBA observations have shown that the slower proper motions measured by the VLA are unlikely to be due to resolution effects.

Despite the aim of this set of observations being to cover a single outburst over a large range of angular scales, the higher-resolution observations could not be triggered early enough to observe the VLA knots and we were unable to conclusively resolve the discrepancy in the proper motions. To do so, VLBA and MERLIN observations should begin within one or two days of the detection of the outburst, to track the knots on the smallest angular scales while they are still bright, and those same knots should be tracked with the A-configuration VLA as they move outwards. If the accretion disc in GRS 1915+105 is indeed now almost empty, then the source may soon switch off 
[14]. There may therefore be few opportunities left to initiate such a monitoring programme, since it requires a bright outburst to occur while the VLA is in its A configuration.

\section{Acknowledgments}

The National Radio Astronomy Observatory is a facility of the National Science Foundation operated under cooperative agreement by Associated Universities, Inc. The X-ray data presented are quick-look results provided by the ASM/RXTE team. MERLIN is operated as a National Facility by the University of Manchester at Jodrell Bank Observatory on behalf of the Particle Physics and Astronomy Research Council (PPARC). This article is an abridged version of a paper submitted to Monthly Notices of the Royal Astronomical Society.

\section{References}

[1] R. D. Blandford and A. Konigl, Relativistic jets as compact radio sources, ApJ 232, 34-48 (1979).

[2] K. M. Blundell and M. G. Bowler, Jet Velocity in SS 433: Its Anticorrelation with Precession-Cone Angle and Dependence on Orbital Phase, ApJ 622, L129-L132 (2005) [astro-ph/ 0410457 ].

[3] A. J. Castro-Tirado, S. Brandt, and N. Lund, GRS 1915+105, IAU Circ. 5590, 2 (1992).

[4] V. Dhawan, I. F. Mirabel, and L. F. Rodríguez, AU-Scale Synchrotron Jets and Superluminal Ejecta in GRS 1915+105, ApJ 543, 373-385 (2000) [astro-ph/ 0006086 ].

[5] R. P. Fender, T. M. Belloni, and E. Gallo, Towards a unified model for black hole X-ray binary jets, MNRAS 355, 1105-1118 (2004) [astro-ph/0409360].

[6] R. P. Fender, S. T. Garrington, D. J. McKay, T. W. B. Muxlow, G. G. Pooley, R. E. Spencer, A. M. Stirling, and E. B. Waltman, MERLIN observations of relativistic ejections from GRS 1915+105, MNRAS 304, 865-876 (1999) [astro-ph/9812150].

[7] R. P. Fender, D. Rayner, D. G. McCormick, T. W. B. Muxlow, G. G. Pooley, R. J. Sault, and R. E. Spencer, Variable circular polarization associated with relativistic ejections from GRS $1915+105$, MNRAS 336, 39-46 (2002) [astro-ph/0204442].

[8] R. M. Hjellming and M. P. Rupen, Episodic Ejection of Relativistic Jets by the X-Ray Transient GRO J1655-40, Nature 375, 464-468 (1995).

[9] P. Kaaret, S. Corbel, J. A. Tomsick, R. Fender, J. M. Miller, J. A. Orosz, A. K. Tzioumis, and R. Wijnands, X-Ray Emission from the Jets of XTE J1550-564, ApJ 582, 945-953 (2003) [astro-ph/0210401].

[10] J. C. A. Miller-Jones, D. G. McCormick, R. P. Fender, R. E. Spencer, T. W. B. Muxlow, and G. G. Pooley, Multiple relativistic outbursts of GRS1915+105: radio emission and internal shocks, MNRAS 363, 867-881 (2005) [astro-ph/0508230].

[11] J. C. A. Miller-Jones, M. P. Rupen, S. A. Trushkin, G. G. Pooley, and R. P. Fender, Radio imaging campaign on newly-detected jets in GRS1915+105, The Astronomer's Telegram 758, 1 (2006).

[12] I. F. Mirabel and L. F. Rodriguez, A Superluminal Source in the Galaxy, Nature 371, 46-48 (1994).

[13] L. F. Rodríguez and I. F. Mirabel, Repeated Relativistic Ejections in GRS 1915+105, ApJ 511, 398-404 (1999) [astro-ph/9808341].

[14] M. Truss and C. Done, The decline and fall of GRS 1915+105: the end is nigh?, MNRAS 368, L25-L29 (2006) [astro-ph/ 0601364 ]. 